\title{
Naturgeschichte und wissenschaftliche Revolution
}

Staffan Müller-Wille

Barrera-Osorio, Antonio, 2006. Experiencing Nature. The Spanish American Empire and the Early Scientific Revolution. Austin: University of Texas Press, geb. 211 S., 45 \$, ISBN-13: 978-0292709812.

Cook, Harold J., 2007. Matters of Exchange. Commerce, Medicine, and Science in the Dutch Golden Age. New Haven/London: Yale University Press, geb. xiv+562 S., 25 f, ISBN-13: 978-0300117967.

Cooper, Alix, 2007. Inventing the Indigenous. Local Knowledge and Natural History in Early Modern Europe. Cambridge: Cambridge University Press, geb. xiv+218 S., 47 f, ISBN-13: 978-0521870870.

Ogilvie, Brian, 2006. The Science of Describing. Natural History in Renaissance Europe. Chicago/London: The University of Chicago Press, geb. 431 S., 45 \$, ISBN-13: 978-0226620886.

Die wissenschaftliche Revolution ist gehörig in Verruf geraten. In ihrer Einleitung zu dem Band zur Wissenschaft in der frühen Neuzeit der Cambridge History of Science konstatieren Lorraine Daston und Katherine Park, dass „traditionelle Behauptungen über die wissenschaftliche Revolution als Ursprung der modernen Welt (oder auch nur moderner Wissenschaft) nicht mehr überzeugen“ (Park/Daston 2006: 13; meine Übersetzung, ebenso die folgenden). Allerdings lassen die Autorinnen ausdrücklich offen, ob die wissenschaftliche Revolution sich nach allem nicht doch auch anders begreifen ließe: weniger vielleicht als epochemachendes Ereignis, in Analogie zur fran- 
zösischen Revolution, denn als eine epochale Umwälzung in der Bewertung und Organisation gesellschaftlichen Wissens, in Analogie also zur industriellen Revolution, die ihr auf lange Sicht immerhin auf dem Fuße folgte. In diesem Sinne lässt sich für die Neuzeit in der Tat ein wichtiger Trend feststellen, der ein Wissensfeld betrifft, das von der Wissenschaftsgeschichte lange vernachlässigt worden ist, die Naturgeschichte nämlich. Sie erfuhr, zunächst im Rahmen der materia medica, eine zunehmende Aufwertung als Garant einer durch Erfahrung und eigene Anschauung statt durch Schlussfolgerung und Autorität geleiteten Wissenschaft.

Der prominenteste Fürsprecher dieser Aufwertung war der englische Rechtsgelehrte und Staatsmann Francis Bacon (1561-1621). Allerdings ließ er sie nicht für Naturgeschichten in jeder beliebigen Form gelten. Eine bloß "narrative“ Naturgeschichte, wie er sie nannte, verglich Bacon mit einem „Königreich, das sich in seinen Angelegenheiten und Geschäften von Straßengerüchten, und nicht von Briefen und Berichten vertrauenswürdiger Kuriere und Botschafter leiten läßt" (1. Bd., S. 4, 94). Eine „induktive“ Naturgeschichte hing von Infrastrukturen der Informationsverarbeitung ab, Formen des „intelligencing“, wie es zeitgenössisch hieß, die vom Arrangement von Schrift- und Bildelementen auf dem Papier über den Aufbau von Korrespondenznetzwerken bis zur Einrichtung von zentralen Institutionen wie Bibliotheken, botanischen Gärten und Laboratorien reichten. Ein solches Unternehmen - ein wahrhaft „königliches Werk“, wie Bacon meinte - konnte nur kollektiv und über lange Zeiträume realisiert werden, wobei keineswegs von vorneherein feststand, welche Techniken und Strategien erfolgreich sein würden. Die Geschichte der Naturgeschichte spielte sich in der Entwicklung von Medien der Informationsverarbeitung sowie im Aufund Ausbau von Netzwerken eines verlässlichen Informationsaustauschs ab (vgl. Dauser/Hächler/Kempe/Mauelshagen/Stuber 2008), und in beiden Hinsichten durchlebte die frühe Neuzeit mit der Erfindung des Buchdrucks und der Globalisierung des Handels enorme Umwälzungen.

Nachdem seit Mitte der 1990er Jahre bereits mehrere Sammelbände erschienen sind, in denen auf diese Zusammenhänge aufmerksam gemacht wurde (Jardine/Secord/Spary 1996, Smith/Findlen 2001, Schiebinger/Swan 2005), liegen aus den vergangenen drei Jahren nun auch eine Reihe von Monographien vor. Sie werfen neues Licht auf die verwirrende, sich oft in abenteuerliche Biographien und minutiöse Einzelheiten verlaufende Geschichte der frühneuzeitlichen Naturgeschichte und dürften auf lange Sicht zu einer endgültigen Dezentrierung des bisherigen Bildes von der wissenschaftlichen Revolution führen. So wendet sich Antonio Barrera-Osorio einer Institution zu, die in der Wissenschaftsgeschichte bislang überhaupt nicht oder allenfalls als randständig wahrgenommen wurde: die Casa de la Contratación (Haus des Handels), die 1503 von der spanischen Krone in Sevilla eingerichtet wurde, um die Handelsbeziehungen mit der Neuen Welt zu organisie- 
ren (S. 14). Eine ganz ähnliche Institution, mit dem bezeichnenden Namen Casa da Índia e Mina, war bereits fünf Jahre zuvor - also bloß sechs Jahre nach Christopher Columbus' Landung in der Karibik - in Lissabon gegründet worden und diente als Vorbild (S. 32-35). 1524 wurde der Casa de la Contratación noch der Consejo de Indias zur Seite gestellt, der sich auf juristische und administrative Aspekte in Bezug auf die neuen Besitzungen der spanischen Krone in Übersee konzentrierte.

Ursprünglich bestand die zentrale Aufgabe der Casa de la Contratación in der Entsendung von Handelsflotten und dem Eintreiben von Steuern. Beide Aufgaben erforderten verlässliches Wissen über Seerouten, Geographie, natürliche Ressourcen und Einwohnerschaft in den neuen Gebieten. Einer der Schwerpunkte der Tätigkeit der Casa de la Contratación wurde, neben der Erstellung von Seekarten, daher schon bald das geordnete Sammeln, Prüfen und Weiterreichen solchen Wissens. Barrera-Osorios übersichtlich gegliedertes und knapp gehaltenes Buch liefert ein Panorama der dabei zum Einsatz gekommenen empirischen Verfahren. Zwei Verfahren wirken aus heutiger Sicht besonders progressiv, da sie das heutige Lizenz- und Patentwesen vorwegnehmen (S. 60). Barrera-Osorio diskutiert diese Verfahren ausführlich am Beispiel von Antonio de Villasante, der 1528 von Santo Domingo nach Spanien reiste, um vom Consejo de Indias ein Monopol über den Handel mit einer Reihe pflanzlicher Drogen zu erwerben. Darunter ein „Balsam“, der aus einem in Santo Domingo wachsenden Baum gewonnen wurde und über dessen medizinische Wirksamkeit Villasante von seiner einheimischen Frau erfahren hatte. Der Consejo war bereit, Villasante das Monopol zuzugestehen, allerdings nur im Tausch gegen einen „langen und sehr vollständigen Bericht über den Baum, [...] wie seine Form beschaffen ist, wo er zu finden ist, und welches Verfahren verwendet wird, um [den Balsam] daraus zu gewinnen“ (S. 16). „Balsam“ war eine auch in Europa produzierte, jedoch ausgesprochen rare Droge. Und um die Identität von Villasantes „Balsam“ entspann sich schon bald ein Streit. Ihn suchte die Krone zu schlichten, indem sie Ärzten und Hospitälern anordnete, den neuen „Balsam“ Prüfungen (esperiencias) zu unterziehen (S. 15-23). Ähnliche Lizenz- und Testverfahren kamen bei der Validierung von Artefakten und technischen Verfahren zum Einsatz, die in der Neuen Welt entwickelt wurden (S. 60-65). Auch die Zuteilung von indigenen Arbeitskräften an die spanischen Kolonisten war an die Auflage gebunden, Beschreibungen und Zeichnungen des bearbeiteten Landes, Erhebungen zur Bevölkerungsstruktur und Angaben zu besteuerbaren Gütern zu liefern (S. 82).

Die Casa de la Contratación besaß aber auch ihre eigenen Experten und bildete solche auch aus, sogenannte "Piloten“, die die Navigation auf den nach Übersee entsandten Schiffen übernahmen. 1508 wurde Amerigo Vespucci zum obersten „Piloten“ (piloto mayor) ernannt und mit der Aufgabe betraut, Seekarten zu erstellen, künftige Piloten in Navigation auszubilden 
und Prüfungen abzunehmen. 1523 kam ein „Kosmograph“ hinzu, der für die Entwicklung und Herstellung von Navigationsinstrumenten zuständig war, und 1552 ein weiterer „Kosmograph“, der nun allein für die Ausbildung von „Piloten“ zuständig war (S. 36-38). Ausbildung und abschließende Prüfung verbanden Theorie und Praxis, und die Casa wurde zu einer Institution, in deren Kontext „die Produktion von Wissen dazu diente, menschliche und materielle Ressourcen in Agenten und Instrumente imperialer Herrschaft zu verwandeln" (S. 48). Francisco Hernandez' Expedition nach Mexiko in den Jahren 1571 bis 1577 sowie die Relaciones geográficas de Indias, die in den 1570er Jahren nach Aussenden eines gedruckten, 37 Kapitel umfassenden Fragebogens an Gouverneure und Bürgermeister in der Neuen Welt vom Consejo de Indias zusammengetragen wurden, bildeten den Höhepunkt dieser Produktion (S. 94-98). Ihren Niederschlag fand sie, wie Barrera-Osorio im letzten Kapitel seines Buches nachweist, in den gedruckten Werken von Gonzalo Fernández de Oviedo (Historia natural y general de las Indias, 1535 und 1557) und José de Acosta (Historia natural y moral de las Indias, 1590), für die bereits ein ausgeprägtes Bewusstsein für die kollektive Natur verlässlichen Wissens charakteristisch ist (S. 118).

Die Casa de la Contratación blieb vorerst eine einzigartige, aber durchaus nicht unbeachtete Institution im frühneuzeitlichen Europa, wie Barrera betont (S. 132). Angesichts der Konkurrenz, in der sich die europäischen Großmächte um die Dominanz über Welthandelsrouten und Niederlassungen in Übersee befanden, dürfte dies kaum überraschen. Eine ähnlich zentrale, straff bürokratisch organisierte und staatlich gelenkte Institution schuf allerdings keine von ihnen. Das Bild, welches das übrige Europa in Hinsicht auf empirische Praktiken bot, ist wesentlich diffuser, auch wenn es ähnliche Elemente wie in Spanien aufweist. Harold J. Cook geht diesen Elementen in aller Ausführlichkeit für die Niederlande in ihrem sogenannten „Goldenen Zeitalter" nach, also in den Jahren, die zwischen der Unabhängigkeit der sieben nördlichen Provinzen von den Habsburgern in den 1580er Jahren und dem Ende des 17. Jahrhunderts liegen. Cook spannt einen weiteren, kulturgeschichtlichen Bogen als Barrera-Osorio, indem er im Anschluss an Georg Simmel die Frage aufwirft, welches kreative Potential die „Informationsökonomie“, das heißt der Austausch von Worten, Dingen und Personen, der die Niederlande mit dem Rest der Welt verband, freisetzte (S. 42-49). Cooks Antwort auf diese Frage fällt unmissverständlich aus: Nichts weniger als „die sogenannte wissenschaftliche Revolution“, hält er am Ende des zweiten Kapitels fest, resultierte aus den globalen Bewegungen von Waren, Nachrichten und Personen und den damit verbundenen „zahllosen Bemühungen [...] sicherzustellen, ob Informationen zutreffend und kommensurabel waren“. „Objektivität“, so Cook weiter, „hatte die Macht, Appetit zu wecken, ja sogar Wahrnehmungen, Begriffe und moralische Bedenken zu verändern. Sie schwebte nicht über der Welt, sondern war tief mit ihr verstrickt." (S. 81) 
Cook belegt dies in einer Reihe von thematisch und chronologisch geschlossenen, relativ locker aneinander gereihten Kapiteln, die eine große Bandbreite von religions-, philosophie-, wissenschafts- und technikhistorischen Themen mit einem gemeinsamen Fluchtpunkt in der Medizingeschichte abdecken. So legt er in Kapitel 3 anhand der Biographie des Botanikers Carolus Clusius dar, dass der niederländische Humanismus nicht so sehr an die eine oder andere Konfession gebunden war, sondern vielmehr an die Fähigkeit, sich der Herrschaft religiöser Dogmen zu entziehen (S. 84) und sich als "Mann von Welt" relativ frei in unterschiedlichsten Kontexten zu bewegen (S. 131). In Kapitel sechs widmet sich Cook der empiristischen Wende im Spätwerk René Descartes' - „einem der bedeutendsten niederländischen Philosophen seiner Zeit", wie er süffisant bemerkt (S. 227) -, und im zehnten Kapitel behandelt er Hermann Boerhaaves Iatromechanismus im Wesentlichen als deskriptives statt theoretisches Unternehmen. In den übrigen Kapiteln hält sich Cook enger an das eigentliche Thema von Matters of Exchange, die Konsonanz zwischen der "neuen Philosophie“ und den "Werten, die im Handel eingebettet waren" (S. 410), indem er auf die hart umkämpften „medizinischen Märkte“ in den städtischen Zentren der Niederlande, insbesondere aber auf deren komplexe Translationsbeziehungen in den ostasiatischen Raum eingeht. Dabei kamen ganz ähnliche empirische Praktiken ins Spiel, wie sie Barrera-Osario für die Casa de la Contratación beschreibt, wenn auch in einem wesentlich diffuseren und konfliktreicheren Feld disparater Institutionen und Kontaktzonen.

Besonders faszinierende Analysen solcher Praktiken bietet Cook mit seinen Fallstudien zu Jakobus Bontius, der 1627 bis 1631 im Auftrag der Vereinigten Ostindischen Compagnie (VOC) das Medizinalwesen in Batavia (heute Jakarta) überwachte und dort nosographische, diätetische und naturhistorische Manuskripte zusammenstellte, die eine Vielzahl von lokalen, schriftlichen wie mündlichen Quellen vereinten (S. 191-209); zu Jan Swammerdam, dessen anatomische, mikroskopische und chemische Studien von dem Versuch angestoßen wurden, ein von Louis de Bils in den späten 1650er Jahren entwickeltes, aber noch erfolgreich geheim gehaltenes Verfahren zur Konservierung anatomischer Präparate offen zu legen und weiter zu entwickeln (S. 276-292); zu Hendrik Adriaan van Reede tot Drakenstein, Kommandant einer Reihe von holländischen Handelsniederlassungen entlang der Südwestküste des indischen Subkontinents, der Mitte der 1670er Jahre ein regelrechtes Komitee - zusammengesetzt aus europäischen und indigenen Experten, Klerikern, Kaufleuten, Apothekern, Ärzten und Brahmanen - zusammenstellte, um die lokale Flora systematisch zu explorieren (S. 310-317); sowie schließlich zu Willem ten Rhijne, der 1674 auf ausdrücklichen Wunsch des japanischen Kaisers hin von der VOC nach Deshima entsandt wurde, um japanische Ärzte und Gelehrte über europäische Heilkunde zu unterrichten, und der im Gegenzug nach seiner Rückkehr aus Japan europäische Gelehr- 
te mit den diagnostischen (Pulsnehmen) und therapeutischen Techniken (Akupunktur, Moxibustion) der chinesischen Medizin vertraut machte (S. 349-377). Was in diesen Fallstudien deutlich wird, ist nicht nur die Intensität - und gelegentlich menschenverachtende Brutalität -, mit der um die Vorherrschaft im Handel mit „Kuriositäten“ und Exotika gekämpft wurde, sondern auch die daraus resultierende Tendenz, den Diskurs auf "Tatsachen“ (matters of fact) zu reduzieren. „Viele Dinge gingen von einer Sprache zur anderen über“, schreibt Cook zusammenfassend, "aber sie neigten dazu, zur Welt der Objektivität zu gehören: schlichte Hauptworte, Adjektive und Verben, die das bezeichnen, was die Sinne uns berichten." (S. 377)

Wenn es soweit den Anschein haben könnte, als sei es insbesondere und ausschließlich der Fernhandel gewesen, der diese "Welt der Objektivität" ins Leben rief, so belehrt uns Alix Cooper eines Besseren. Sie beginnt ihr Buch mit einer vielleicht überraschenden, sprachgeschichtlichen Tatsache, nämlich dass Ausdrücke wie „eingeboren“ oder „indigen“ im Europa der frühen Neuzeit nicht etwa auf außereuropäisches Bezug nahmen, sondern vielmehr einem „nach innen“ gerichteten Blick entsprangen. In einer Art Gegenbewegung zu den von Barrera-Osorio und Cook beschriebenen Handels- und Verkehrsströmen wandten sich Mediziner und Botaniker der Erforschung naheliegenderer, heimischer Ressourcen zu, insbesondere in Regionen wie dem deutschsprachigen Raum, die aufgrund ihrer kontinentalen Lage nur aus zweiter Hand an diesen Strömen partizipieren konnten. Ideologisch wurde diese Gegenbewegung von Überzeugungen getragen, die eine enge Kopplung von geographischem Raum, spezifischen Krankheiten und einheimischen Heilmitteln behaupteten, polemisch die Nützlichkeit importierter Drogen bestritten und Autarkie als wirtschaftspolitisch erstrebenswertes Ziel bezeichneten. Insbesondere Paracelsisten machten sich in dieser Hinsicht lautstark bemerkbar (S. 29-31), ähnliche Ansichten finden sich aber auch bei Humanisten wie Hieronymus Bock, Symphorien Champier, Robert Burton und Jan van Beverwyck (S. 35-45). Coopers groteske Überzeichnung Carl von Linnés als Botaniker, der am Ende der von ihr betrachteten Periode „die Beiträge zur lokalen Naturgeschichte stark entwertete“ (S. 167), deutet darauf hin, dass es sich bei der Akzentuierung des Gegensatzes von lokalem und globalem Naturwissen oft nur um eine rhetorische Taktik gehandelt haben dürfte, denn gerade Linné ging, wie Cooper eingesteht (S. 170), von einer Vereinbarkeit der beiden Pole aus. Dies sollte aber nicht davon ablenken, dass sich parallel zur Erforschung außereuropäischer Floren und Faunen auch die Perspektive auf europäische Naturräume in der frühen Neuzeit radikal verschob.

Cooper zeigt dies, indem sie mit großer Aufmerksamkeit für buchhistorische Details die Entstehungsgeschichte von neuartigen Genres in der naturhistorischen Literatur nachzeichnet. Da sind zunächst die im frühen 17. Jahrhundert vor allem im deutschsprachigen Raum einsetzenden Pu- 
blikationen von lokalen Floren zu nennen, die sich auf die Aufzählung der Pflanzenarten beschränkten, die in den Gärten und im näheren Umkreis einer Universitätsstadt anzutreffen waren (S. 55). Von handlichem und schon bald standardisiertem, auf Nennung, kurze Diagnose, Standort und gegebenenfalls Nutzen reduziertem Format, begleiteten diese Lokalfloren als regelrechte „Taschenbücher“ (S. 73) Studenten und Lehrer der materia medica auf ihren Exkursionen, wie erhalten gebliebene, durchschossene und reich annotierte Exemplare beweisen (S. 75). Bereits 1727 war beispielsweise die Flora um Altdorf in einem halben Dutzend solcher Kataloge dokumentiert, während dieses Format erst gegen Ende des 17. Jahrhunderts auch in exotische Regionen übertragen wurde, etwa mit der Flora Jamaicensis, die Hans Sloane 1696 veröffentlichte (S. 71f.).

Neben dieser Transposition des Modells der Lokalflora in koloniale Kontexte beobachtet Cooper aber auch seine Ausweitung am Ende des 17. Jahrhunderts auf regionale und territoriale Naturgeschichten innerhalb Europas. Erstere untersucht sie am Beispiel von Veröffentlichungen, die im Schnittfeld von Kameralismus und Naturtheologie Bestandsaufnahmen der Bodenschätze einer bestimmten Region lieferten, letztere vor allem im Werk des Schweizer Naturhistorikers Johann Jakob Scheuchzer. Naturgemäß waren diese Unternehmen in weit größerem Maße auf den Austausch zwischen Sammlern, Korrespondenz, umfangreiche bibliographische Recherchen und systematische Umfragen angewiesen. Wie wenig von vorneherein feststand, welche Form gerade letztere nehmen sollten, zeigt sich am Beispiel Henry Oldenbourgs, der seinen Korrespondenten im Gegensatz zu Scheuchzer Auskünfte abverlangte, die zwar sehr spezifische Gegenstände betrafen, aber gleich für ganze Territorien abgegeben werden sollten (S. 122). Scheuchzer dagegen zeigte bei seinen Umfragen, vor allem aufgrund seiner bibliographischen Studien, sehr viel mehr Sensibilität für die „Geopolitik der Wissenschaft" (S. 163). Das anscheinend "lokale“ Wissen, das sich in den von Cooper behandelten Werken niederschlug, war in Wahrheit „lokalisiertes“ Wissen (S. 86) - ein Wissen, welches zwar aus lokalen Quellen geschöpft wurde, aber doch in einem translokalen Netz von Literaturverweisen und Austauschbeziehungen eindeutig „verortet“ wurde.

Diesen Gesichtspunkt macht sich Brian Ogilvie zu eigen. Seiner Auffassung nach nahm die Naturgeschichte bereits im Laufe der Renaissance die Züge einer „distinkten Disziplin“ (S. 2) an, nachdem sie zuvor allenfalls als Hilfswissenschaft von Medizin und Naturphilosophie gegolten hatte. Dabei macht er aus seiner Ablehnung von Interpretationen, die die frühmoderne Naturgeschichte in den Kontext von Patronage und "protokapitalistischen" Tauschsystemen stellen, keinen Hehl (S. 13f.). Man kann ihm in diesem Punkt vielleicht vorwerfen, symbolischen mit kommerziellem Tausch zu verwechseln - Carolus Clusius' offenbarer Verwicklung in den Tulpenhandel gegen Ende des 16. Jahrhunderts begegnet er beispielsweise mit dem Hinweis, dass 
"Geld keinen Platz im Tausch [zwischen Botanikern] hatte“ (S. 78). Ogilvies Frage nach der spezifischen „kulturellen Form“ der frühmodernen Naturgeschichte bleibt für ein Verständnis der wissenschaftlichen Revolution jedoch relevant, da das, was dieser Form Identität gab, gerade die „Auseinandersetzung um Methoden der Kommunikation und Reproduktion von Naturerfahrung" war (S. 15). Seine internalistische Perspektive fokussiert den Blick auf Probleme der Beschreibung, Kollation und Translation, die sich auch außerhalb der Disziplin Naturgeschichte stellten, aber innerhalb derselben geradezu zum identitätsstiftenden, epistemischen Objekt einer Wissenschaft wurden (S. 7f.).

Ogilvie gliedert seine Darstellung nach vier Generationen, beginnend mit den italienischen Ärzten und Humanisten, die sich mit Ausgaben antiker Texte zur Botanik beschäftigten (1490 bis 1530), gefolgt von den meist aus Nordeuropa stammenden Verfassern früher Kräuterbücher (1530-1560), einer weiteren Generation, die sich in die aufblühende Sammlungskultur einschrieb (1560-1590), und schließlich einer vierten Generation, die sich angesichts der zu Tage tretenden Vielfalt von Naturobjekten mehr und mehr Fragen der Nomenklatur und Taxonomie zuwandte (1590-1620). Allerdings gibt er dieser relativ konventionellen Gliederung eine entscheidende Wendung, indem er jede dieser Generationen mit der kritischen Fortschreibung, der Korrektur und Ergänzung der Werke der vorangehenden Generation beschäftigt sieht. Für die erste Generation hatte noch die Identifikation der von Dioscurides und Plinius erwähnten Pflanzen im Vordergrund gestanden, aber bereits 1492 trat Niccoló Leoniceno mit einem Buch über die zahlreichen "Irrtümer“, die er vor allem Plinus unterstellen zu können glaubte, eine Debatte über die Vertrauenswürdigkeit antiker Quellen los (S. 126-131). Und die zweite Generation, die überwiegend an den oberitalienischen Universitäten studiert hatte und das dort erworbene Wissen nun an nördlicher gelegene Universitäten trug, bezog ihren Antrieb vor allem aus der Tatsache, dass viele mitteleuropäische Pflanzenarten ihren Lehrern unbekannt geblieben waren (S. 137).

Wie bei Barrera-Osorio, Cook und Cooper rücken so auch für Ogilvie die geographische und kollektive Dimension der frühneuzeitlichen Naturgeschichte in den Blickpunkt sowie die Techniken, die zur Überbrückung von räumlichen und zeitlichen Distanzen entwickelt wurden (S. 52-63). Seine Geographie bleibt allerdings auf die "Gelehrtenrepublik“ (republic of letters) Europas beschränkt, die sich im offenen Gegensatz zur höfischen und städtischen Kultur definierte (S. 58, 85). Spanische und portugiesische Gelehrte bleiben damit außen vor (S. 55), und Clusius erscheint mit seinen Reisen nach Wien und Spanien als ein "Odysseus des Hinterlands“ (S. 143). Der Vorteil dieser Beschränkung liegt darin, dass sich Ogilvie mit großer Zielgenauigkeit an die Untersuchung der empirischen Verfahren machen kann, die Botaniker des 17. Jahrhunderts im engen Austausche miteinander entwi- 
ckelten. Da ist an vorderster Stelle das Herbarium zu nennen, das als Unterrichtsmittel bereits Mitte des 16. Jahrhunderts verwendet wurde und sich im Laufe des 17. Jahrhunderts zu einem regelrechten Repositorium botanischen Wissens entwickelte, indem es mit Pflanzenzeichnungen integriert und als Projektionsfläche für laufende Annotationen verwendet wurde (S. 165-174). Daneben experimentierten Naturhistoriker mit einer Vielfalt von Aufzeichnungs- und Exzerptverfahren, um Gelesenes und brieflich Mitgeteiltes nicht nur aufzubewahren, sondern auch neu zu arrangieren (S. 174-182).

Aus solchen literarischen Techniken gingen im Druck Beschreibungen und Abbildungen hervor, die sich zum einen eng und mit standardisierter Terminologie und Gliederung an der Morphologie des Objekts orientierten - Ogilvie spricht von „stellvertretenden“ (vicarious) Beschreibungen, insofern sie den Leser glauben lassen, das Objekt selbst vor Augen zu haben (S. 183) -, zum anderen aber aus der Kollation einer Vielzahl von eigenen Beobachtungen und Beobachtungen anderer hervorgingen. Die Spannung zwischen diesen beiden Polen thematisiert Ogilvie am Ende seines Buches, wenn er die Verfahren diskutiert, die Konrad Gessner, Ulysse Aldrovandi und Carolus Clusius verwendeten, um durch Unterscheidung von abhängigen und unabhängigen Quellen die Vertrauenswürdigkeit von naturhistorischen Berichten zu überprüfen. Ironischerweise zeigen sich in diesen Fallstudien nicht nur die Grenzen der frühmodernen Naturgeschichte (S. 236), sondern auch die von Ogilvies eigenem Ansatz - soweit es die Tierwelt des Nordens anging, insbesondere das Walross, setzte Conrad Gessner offenbar viel Vertrauen in den Portugiesen Damião da Goes, der sich im 16. Jahrhundert immerhin selbst in Skandinavien aufgehalten hatte (S. 237); und das letzte Wort in der Frage, ob Paradiesvögel Beine haben oder nicht, behielten für Clusius Antonio Pigafetta, der Ferdinand Magellan auf dessen erster Weltumseglung begleitet hatte (1519-1522), und die Seeleute, die Bälge von Paradiesvögeln nach Europa einführten (S. 251).

Die gelehrte Naturgeschichte scheint also trotz allem durchlässiger für die zahllosen, globalen Bewegungen von Personen und Dingen gewesen zu sein, die vor allem Cook so lebendig beschreibt und es spricht vieles dafür, dass die disziplinäre Eigendynamik, die sie in der frühen Neuzeit gewann, nur vor diesem bewegten Hintergrund zu verstehen ist. Paula Findlen behauptet, dass die massive Aufwertung, welche die Erfahrung aus eigener Anschauung zu Beginn des 16. Jahrhunderts erfuhr, paradoxerweise darauf zurückzuführen ist, dass mehr und genauer gelesen wurde (Findlen 2006: 442). Sowohl Barrera-Osorio (S. 8, 11) als auch Cook (S. 83f.) wenden sich ausdrücklich gegen die von Max Weber und Robert K. Merton aufgestellte These, dass Frühformen des Kapitalismus und der modernen Wissenschaft in ursächlichem Zusammenhang mit einer protestantischen Ethik standen. Trifft das von ihnen, aber auch von Cooper und Ogilvie gezeichnete Bild zu - und zieht man darüber hinaus noch neuere Arbeiten hinzu, welche die Be- 
deutung des atlantischen Raums für die Literatur- und Kunstgeschichte der alten wie neuen Welt betonen (Phaf-Rheinberger 2008) -, so steht die Wissenschaftsgeschichte unverhofft vor dem Problem, dass die wissenschaftliche Revolution nicht mehr auf einen plötzlichen Wandel in Weltbildern und Mentalitäten zurückzuführen ist, sondern als ein kumulativer und kollektiver Prozess verstanden werden muss, in dem Verfahren der Fixierung, Verarbeitung und Distribution von Erfahrungswissen ein zuvor nicht dagewesenes, Grenzen und Traditionen überschreitendes Spiel von Überprüfung und Rückfrage, Ergänzung und Berichtigung ermöglichten.

\section{Literatur}

Dauser, Regina/Hächler, Stefan/Kempe, Michael/Mauelshagen, Franz/Stuber, Martin, Hg., 2008. Wissen im Netz. Botanik und Pflanzentransfer in europäischen Korrespondenznetzen des 18. Jahrhunderts. Berlin: Akademie Verlag (=Colloquia Augustana 24).

Findlen, Paula, 2006. Natural History. In: Katharine Park und Lorraine Daston, Hg., Early Modern Science. Cambridge: Cambridge University Press (=The Cambridge History of Science, 3), 435-468.

Jardine, Nick/Secord, James A./Spary, Emma C., Hg., 1996. Cultures of Natural History. Cambridge: Cambridge University Press.

Park, Katherine/Daston, Lorraine, 2006. Introduction: The Age of the New. In: Katharine Park und Lorraine Daston, Hg., Early Modern Science. Cambridge: Cambridge University Press (=The Cambridge History of Science, 3), 1-17.

Phaf-Rheinberger, Ineke. 2008. The Air of Liberty. Narratives of the South Atlantic Past. Amsterdam: Rodopi.

Smith, Pamela/Findlen, Paula, Hg., 2001. Merchants and Marvels: Commerce, Science, and Art in Early Modern Europe. London: Routledge.

Schiebinger, Londa/Swan, Claudia, Hg., 2005. Colonial Botany. Science, Commerce, and Politics in the Early Modern World. Philadelphia: University of Pennsylvania Press.

Staffan Müller-Wille

ESRC Centre for Genomics in Society

University of Exeter

Byrne House, St Germans Road

Exeter, Devon EX4 4PJ

Großbritannien

E-Mail: S.E.W.Mueller-Wille@exeter.ac.uk 